\title{
Frequency and Temperature Dependence of Electrical Breakdown at 21, 30, and $39 \mathrm{GHz}$
}

\author{
H. H. Braun, S. Döbert, I. Wilson, and W. Wuensch* \\ European Organization for Nuclear Research (CERN), CH 1211 Geneva 23, Switzerland
}

(Received 1 July 2002; published 5 June 2003)

\begin{abstract}
A TeV-range $e^{+} e^{-}$linear collider has emerged as one of the most promising candidates to extend the high energy frontier of experimental elementary particle physics. A high accelerating gradient for such a collider is desirable to limit its overall length. Accelerating gradient is mainly limited by electrical breakdown, and it has been generally assumed that this limit increases with increasing frequency for normal-conducting accelerating structures. Since the choice of frequency has a profound influence on the design of a linear collider, the frequency dependence of breakdown has been measured using six exactly scaled single-cell cavities at 21,30 , and $39 \mathrm{GHz}$. The influence of temperature on breakdown behavior was also investigated. The maximum obtainable surface fields were found to be in the range of 300 to $400 \mathrm{MV} / \mathrm{m}$ for copper, with no significant dependence on either frequency or temperature.
\end{abstract}

DOI: 10.1103/PhysRevLett.90.224801

PACS numbers: 29.17.+w, 41.75.Lx, 52.80.Pi, 52.80.Vp

Introduction.-The feasibility of a compact $e^{+} e^{-}$ linear collider (CLIC) [1] which aims for a center-ofmass energy in the $\mathrm{TeV}$ range is studied at CERN within an international collaboration. CLIC is characterized by the choice of a very high accelerating gradient of $150 \mathrm{MV} / \mathrm{m}$, a high operating frequency of $30 \mathrm{GHz}$, and a two-beam accelerator scheme to produce the necessary rf power. The high-power testing of rf structures is currently being carried out in the CLIC Test Facility (CTF II) [2], a two-beam accelerator providing up to $280 \mathrm{MW}$ of $30 \mathrm{GHz}$ power at a pulse length of $16 \mathrm{~ns}$. The discovery two years ago of substantial damage due to electrical breakdowns in prototype $30 \mathrm{GHz}$ structures at accelerating fields of about $60 \mathrm{MV} / \mathrm{m}$ obliged the CLIC team to undertake a more systematic study of the phenomenology of $\mathrm{rf}$ breakdown. To complement the rather expensive development and testing of traveling wave CLIC-type accelerating structures, a series of experiments using simple single-cell standing-wave cavities, directly driven by a high-charge electron beam, were performed. This test setup enables very high surface fields to be obtained in the cavity at a well defined location. These tests assume that the maximum electrical field on the surface is the key parameter for breakdown initiation, and enable fundamental questions such as frequency and temperature dependence of the breakdown behavior to be investigated in a relatively simple way.

Cavity design and fabrication.-A total of six cavities, two at each of three different frequencies $(21,30,39 \mathrm{GHz})$ were made. These high-gradient single-cell cavities have a pillbox-type geometry exactly scaled for the different frequencies for operation with the transverse-magnetic $\left(\mathrm{TM}_{010}\right)$ mode (see Fig. 1). The scaling factor $s=$ $30(\mathrm{GHz}) / f_{0}(\mathrm{GHz})$ was applied to the beam-pipe diameter, the cavity length, the cavity diameter, and the radius at the beam-pipe opening. The location of the maximum surface field $\hat{E}_{S}$ in these cavities is also indicated in the figure. A small coupling aperture (about $1 \mathrm{~mm}$ wide) was milled into the outer cavity wall to enable the field in the cavity to be sampled through a piece of standard waveguide. The coupling was chosen sufficiently small to keep the impact on $Q$ negligible. The $Q$ values of the cavities varied by a factor of 1.5 due to both the geometry scaling and the surface resistance change with frequency. For the $30 \mathrm{GHz}$ geometry the cavity length and the waveguide rectangular size (WR) 28 waveguide height are identical, while for 39 and $21 \mathrm{GHz}$ a taper to the standard waveguide height of WR 28 and WR 42, respectively, was used. The cavities were made out of oxygen-free highconductivity copper parts which were brazed together in a vacuum oven at $820^{\circ} \mathrm{C}$. All but one of the cavities were machined on conventional lathes, one of the $30 \mathrm{GHz}$ cavities was, however, machined by single-point diamond turning on a state-of-the-art high-precision lathe which produced an optical surface finish. Besides the standard CERN cleaning process for copper pieces consisting of several steps of degreasing, rinsing with deionized water, and etching lightly, no special surface preparation was performed, either before or after brazing the parts together. The outer wall of the brazed cavities was dimple tuned to the desired operating frequency and the

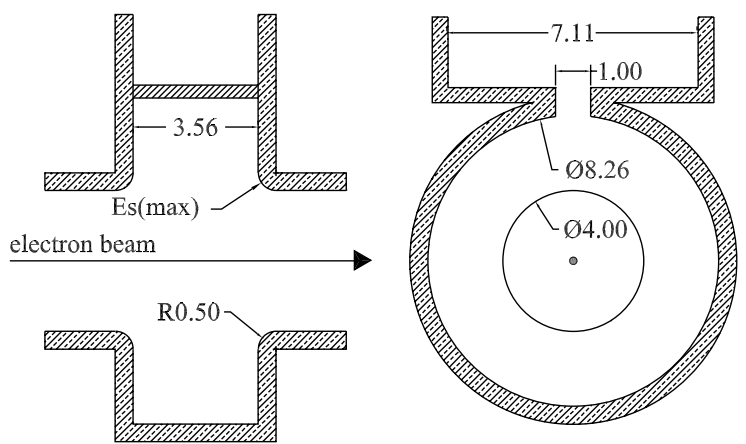

FIG. 1. Geometry of a $30 \mathrm{GHz}$ cavity. Distances in $\mathrm{mm}$. 


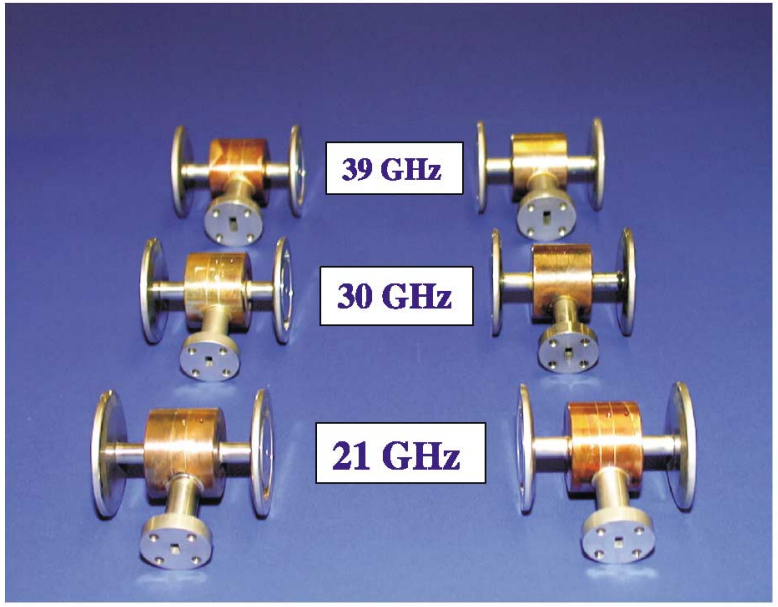

FIG. 2 (color online). Photograph of the six single cell cavities tested.

rf parameters determined using a cold-measurement setup. The six cavities are shown in Fig. 2.

Experimental setup.-After installation in the CTF II test accelerator an in situ bakeout at $120^{\circ} \mathrm{C}$ for $48 \mathrm{~h}$ was performed, resulting in a pressure of the order of $10^{-8}$ mbar. This vacuum degraded by about 1 order of magnitude while running with a high drive-beam charge. The cavities were driven by a $3 \mathrm{GHz}$ electron bunch train consisting of 24 bunches with a charge of up to $11 \mathrm{nC}$ per bunch. Wall current monitors upstream and downstream of the cavity were used to ensure a good transmission of the beam through the cavity without major losses. The main experimental observable in these experiments was the output rf pulse which has a fast rise from the shock excitation with the drive beam, followed by an exponential decay (see Fig. 3). Because of the high $Q$ of the cavities, the decay of the field inside the cavities could be observed for some tens of nanoseconds after the electron beam had passed. The $30 \mathrm{GHz}$ rf signal was mixed down to an intermediate frequency of $500 \mathrm{MHz}$ and then digitized. From these data the envelope of the rf amplitude and time-resolved phase information were determined. An electrical breakdown was indicated by a pulse shortening of the exponential decay, which implies a very fast dissipation of the stored energy in the cavity. Two examples of pulse shortening due to breakdown are shown in Fig. 3 (the long pulse corresponds to the situation of no breakdown). The actual value of the surface field was obtained by measuring the stored energy in the cavity, taking into account the simulated relation between stored energy and maximum surface field. The drivebeam charge needed to obtain the fields was used to cross-check the calibration using the computed $R / Q$ of the cavities. Although most of the breakdowns occurred 8-12 ns after the peak field, some events nevertheless occurred up to $60 \mathrm{~ns}$ after the maximum. The energy was always dissipated in less than $10 \mathrm{~ns}$ and was associated

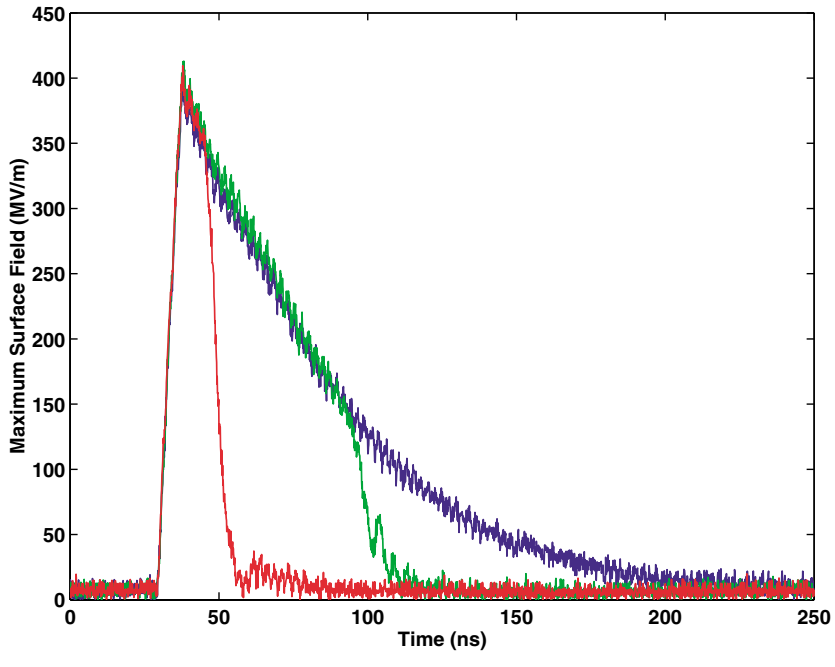

FIG. 3 (color online). Envelope of typical rf pulses with and without pulse shortening from a $30 \mathrm{GHz}$ cavity.

with a frequency shift to higher frequencies. This frequency shift (typically $100 \mathrm{MHz}$ ) could possibly be due to massive electron loading or plasma formation. Details of the experimental aspects can be found in [3].

Frequency dependence.-To obtain a minimum statistical sample, two cavities at each frequency were tested. Each of the cavities was conditioned with about $5 \times 10^{5}$ shots at a repetition rate of $5 \mathrm{~Hz}$. After this number of pulses a clear saturation of the obtainable field was observed. The maximum obtainable surface field was defined as the maximum field level without breakdowns at the end of the conditioning. The field limit in all cases had a well defined threshold. The maximum surface field measured for the different cavities is shown in Fig. 4. The field limitations do not show a significant frequency dependence within the accuracy of the experiment. The error bars shown in the plot include uncertainties in the rf

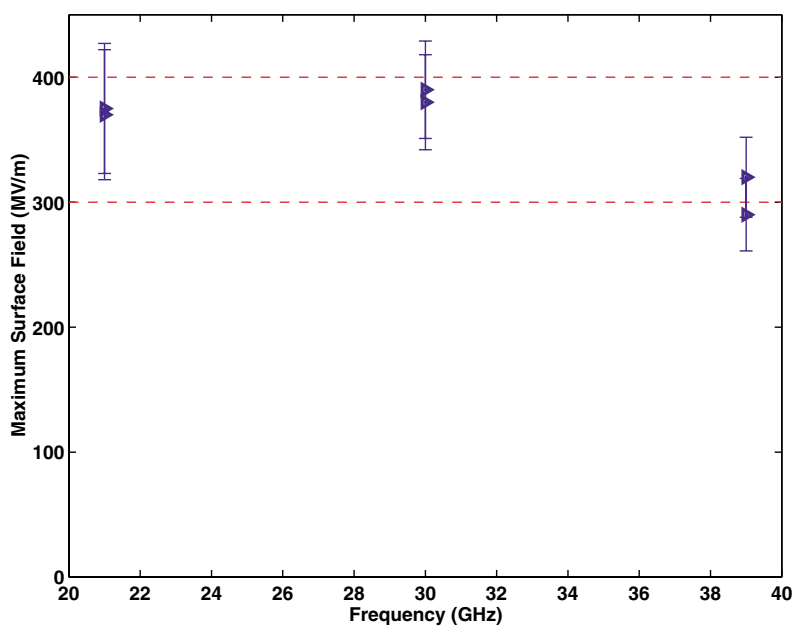

FIG. 4 (color online). Frequency dependence of the maximum surface field. 
calibrations as well as in the geometry of the high field region of the cavities entering into the field calculations. The maximum obtainable surface field is certainly not increasing with frequency, while the $39 \mathrm{GHz}$ results seem to indicate an even lower limit at the highest frequency. The breakdown behavior in terms of the frequency shift and the speed of the energy dissipation was also found to be independent of frequency although the stored energy in the cavities at different frequencies varied by a factor of 8 , and the $Q$ values varied by a factor of 1.5 .

After the measurements were completed, the cavities were cut open and analyzed by scanning electron microscopy. The analysis revealed surface modifications which were concentrated in the high field areas on the beampipe radius. Melted and pitted areas were found as well as copper droplets. The features appeared in all cavities, but the amount of surface modifications were correlated with frequency: the higher the frequency the larger the melted zones. The spatial distribution of the surface melting indicated that its origin was breakdown related. Some influence of the driving beam cannot be totally excluded because the free aperture for the beam was also scaled with frequency and hence correlated with the amount of surface modification. However, the breakdown level was found to depend only on the field level induced in the cavity, and to be independent of beam loss. This was confirmed by intentionally steering the beam into the cavities.

Temperature dependence.-The motivation for measuring the temperature dependence of breakdown was that some models to explain rf breakdown involve a heating process. Examples include explosive electron emission, melting by electron impact, and exploding dust particles. The unexpected observation of delayed breakdowns in the experiments described above also favors a heating process.

The diamond-machined $30 \mathrm{GHz}$ cavity was cooled down with liquid nitrogen and heated up with a hot-air gun, providing a temperature variation of almost $500 \mathrm{~K}$. The cooling was done by attaching a copper block which was directly cooled by liquid nitrogen to an already conditioned cavity. The temperature was calculated using simple thermal expansion considerations from the resonant frequency, which was measured every rf pulse. The surface resistivity of the inside surface of the cavity was determined from the test data by first measuring the loaded $Q$ from the decay curve and assuming that the external $Q$, which is a function of geometry only, remained constant during the temperature cycling. Figure 5 shows a comparison of the surface resistivity measured by this method as a function of temperature, and data from the literature for dc experiments [4]. The maximum surface field as a function of temperature was also found to be constant within the measurement accuracy. Potentially relevant parameters for heating processes, such as the pulse length and instantaneous power

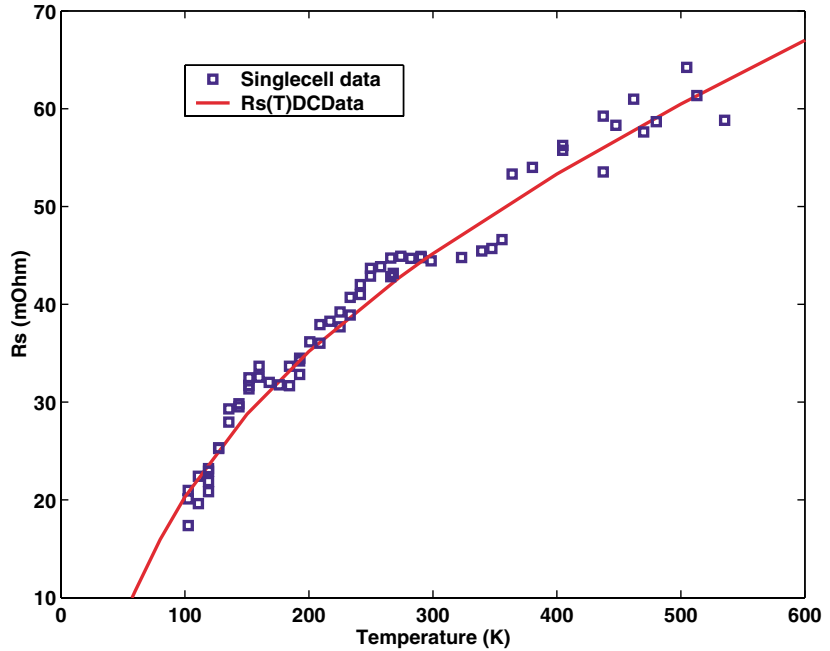

FIG. 5 (color online). Comparison of the measured surface resistivity with literature data of copper.

dissipated at the surface, vary considerably as a function of temperature in all these tests. The variation of the unloaded $Q$ by about a factor of 3 gives a measure of this difference. The temperature dependence of the maximum surface field is plotted in Fig. 6.

Conclusions and discussion.-This experiment is to the authors' knowledge the first to measure geometrically exactly scaled cavities at different frequencies in order to obtain the frequency scaling of the maximum obtainable surface fields. The maximum surface field was found to be limited to between 350 and $400 \mathrm{MV} / \mathrm{m}$ for singlecell copper cavities and to be independent of both frequency and temperature in the measured range. These results contradict the generally accepted belief that higher frequencies enable higher gradients to be attained without breakdowns. Commonly used scaling laws predicted a Kilpatrick-like [5] square-root or at least a cube-root

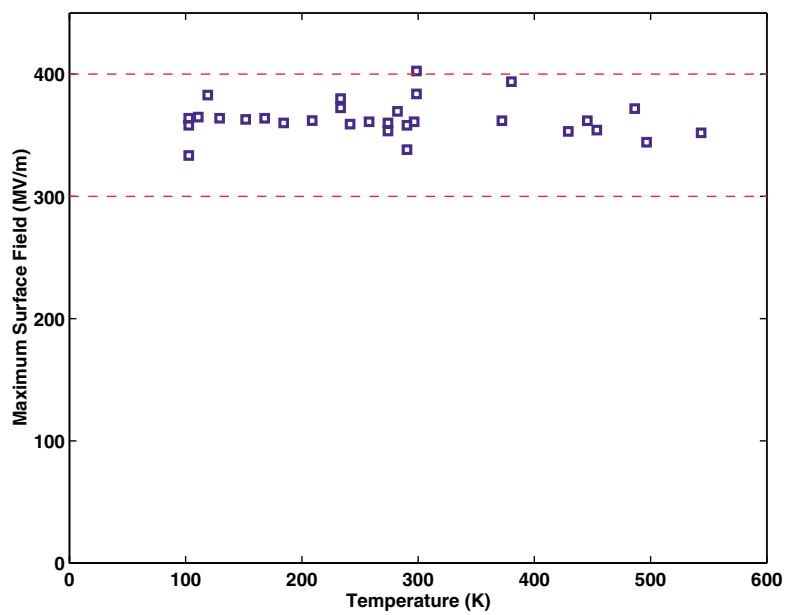

FIG. 6 (color online). Temperature dependence of maximum surface field. 
[6] dependence based on data fits. Although such scaling laws seem to work at lower frequencies, the present results indicate that extrapolation to the $30 \mathrm{GHz}$ range is not justified. One explanation could be that there is a completely different breakdown mechanism at higher frequencies, higher fields, and shorter time scales. Kilpatrick assumed ion acceleration and impact to derive his criterion which is clearly less relevant at very high frequencies where ion mobility is much reduced. A copper ion, for instance, oscillates with an amplitude of $20 \mathrm{~nm}$ in a rf field of $250 \mathrm{MV} / \mathrm{m}$ at $30 \mathrm{GHz}$. The Fowler-Nordheim [7] description for field emission is independent of frequency and is therefore a candidate for the limitations found in this experiment. Indeed, if we take the maximum surface field of $350 \mathrm{MV} / \mathrm{m}$ and multiply it with a typical empirical field enhancement factor $(\beta=30)$ we reach the same limit of about $10 \mathrm{GV} / \mathrm{m}$ found in $\mathrm{dc}$ experiments [8]. Under these conditions the field emitter starts to melt and initiates a breakdown.

From the temperature scanning experiment, one can draw the conclusion that the heating process responsible for the rf breakdowns is extremely fast; otherwise, it would modify the field limits or the temporal behavior of the energy dissipation as a function of the cavity temperature. Explosive electron emission [8] is known to take a few nanoseconds, which matches the present observations. Also the observed frequency shift indicates an electronic process. The scanning electron microscope images of the inner surfaces of the cavities indicate that the impact of the emitted electrons leads also to local melting. The independence of temperature could have supported a multipacting scenario but the independence of frequency and the observation of delayed breakdowns are not consistent with this explanation.

The experimental evidence points to a fundamental limitation of a maximum surface field of around $350 \mathrm{MV} / \mathrm{m}$ on copper surfaces for pulses longer than $10 \mathrm{~ns}$. With a ratio of 2 between the maximum surface field and the actual accelerating field in an accelerating structure, the goal of $150 \mathrm{MV} / \mathrm{m}$ accelerating gradient appears challenging but is not excluded. A possible improvement could be the choice of other materials than copper which better resist electron impact (tungsten, for example) and explosive emission, or simply emit fewer electrons (high work function). An understanding of the mechanism of the sudden energy dissipation is essential.

About the same maximum surface fields were found while testing multicell traveling wave prototype accelerating structures $[9,10]$; therefore the belief is that the single-cell standing-wave results are also applicable and relevant for this work. This assumption was confirmed by the similarity of the surfaces obtained after high-gradient rf processing of traveling wave structures and the described single-cell cavities. It should be noted that a fundamental surface field limit of $350 \mathrm{MV} / \mathrm{m}$ for copper contradicts one previous report of high-gradient tests [6] which obtained a surface field of $445 \mathrm{MV} / \mathrm{m}$ at $C$-band and $572 \mathrm{MV} / \mathrm{m}$ at $X$-band. Unfortunately, no subsequent test has ever achieved similar fields. It would be very desirable, and a very important contribution to the understanding of high-gradient limits, to try to reproduce these results.

Finally, it is important to remember for linear collider design that, although achievable gradient may not increase with frequency, a high frequency is still desirable to minimize the peak input power of accelerating structures which for a given geometry decreases with the inverse square of the frequency.

Thanks are due to C. Achard, D. Allard, and S. Leblanc for their help in building and installing these experiments and to G. Arnau and M. Brandt from the $\mathrm{EST} / \mathrm{SM} / \mathrm{ME}$ group at CERN for the postmortem surface analysis and SEM imaging.

*Electronic address: Steffen.Doebert@cern.ch

[1] The CLIC Study Team, edited by G. Guignard, CERN Report No. 2000-008, 2000.

[2] H. H. Braun et al., in Proceedings of the 7th European Particle Accelerator Conference, Vienna, Austria, 2000 (Austrian Academy of Sciences Press, Vienna, 2000), p. 271.

[3] C. Achard, H. H. Braun, S. Döbert, L. Groening, I. Wilson, and W. Wuensch, CERN CLIC Note 498, 2001.

[4] Handbook of Chemistry and Physics, edited by D. R. Lide (Chemical Rubber Company, Boca Raton, FL, 1999), 80th ed.

[5] W. D. Kilpatrick, Rev. Sci. Instrum. 28, 824 (1957).

[6] G.A. Loew and J.W. Wang, Report No. SLAC-PUB-7684, 1997.

[7] R. H. Fowler and L. Nordheim, Proc. R. Soc. London A 119, 173 (1928).

[8] G. A. Mesyats and D. I. Proskurovsky, Pulsed Electrical Discharge in Vacuum (Springer, Heidelberg, 1989).

[9] H. H. Braun, S. Döbert, L. Groening, I. Wilson, W. Wuensch, and F. Zhou, CERN CLIC Note 475, 2001.

[10] J.W. Wang, G. A. Loew, R. J. Loewen, R. D. Ruth, A. E. Vlieks, I. Wilson, and W. Wuensch, Report No. CERNSL-95-27-RF, 1995. 